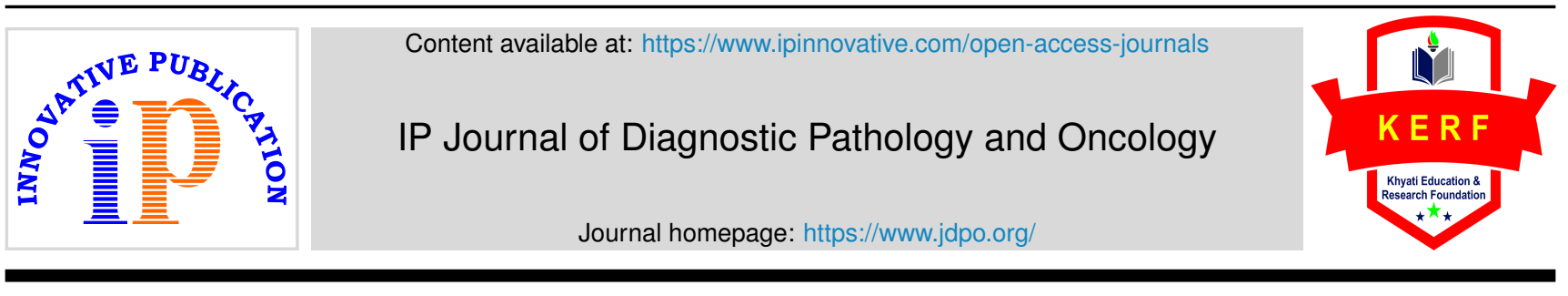

Original Research Article

\title{
A Study of levels of serum Adiponectin, Hemoglobin and RBCs in patients with acute and chronic kidney diseases
}

\author{
Anil Malleshi Betigeri ${ }^{1}$, Uma $\mathbf{K}^{1, *}$ \\ ${ }^{1}$ Dept. of Pathology, Akash Institute of Medical Sciences and Research Centre, Devanahalli, Bangalore, Karnataka, India
}

\section{A R T I C L E I N F O}

\section{Article history:}

Received 28-01-2021

Accepted 03-02-2021

Available online 10-03-2021

\section{Keywords:}

AKI

CKD

Serum Adiponectin

Hemoglobin

RBCs.

\begin{abstract}
A B S T R A C T
Background: Loss or reduced of kidney function leads to acute and chronic kidney diseases. Since past decades onwards kidney diseases showed negative impact on kidney patients in short and long time. For estimating serum adiponectin, Hemoglobin and RBCs levels are useful for early diagnosis and progression of AKI and CKD.

Materials and Methods: This is the case control study was carried out the association of serum Adiponectin levels with blood count in patients with Kidney diseases and healthy controls. A total 150 subjects included in this study in that 50 Acute Kidney Injury patients, 50 Chronic Kidney Injury Patients and 50 healthy controls was included, blood and urine samples was collected from all the included subjects. The serum Adiponectin levels are estimated by ELISA, and HB, RBCs was analysed by CBC Analyser.

Results: The present study evaluates the serum levels of Adiponectin in patients with acute, chronic kidney diseases and compared with the healthy controls. Serum Adiponectin $\left(11.89 \pm 4.95, \mathrm{P}-0.0001^{* *}\right)$ levels more significant elevated in two groups of acute, chronic kidney diseases and compared with the healthy controls. This study also evaluates the hemoglobin and RBCs levels in patients with acute, chronic kidney diseases and compared with the healthy controls. The statistically significant difference between the serum Adiponectin and hemoglobin and RBCs levels in patients with acute, chronic kidney diseases and compared with the healthy controls.

Conclusions: This study suggesting that the serum Adiponectin and hemoglobin and RBCs levels useful for early detection of acute, chronic kidney diseases. To frequent monitoring serum Adiponectin and hemoglobin and RBCs levels may be useful for further complications.
\end{abstract}

(C) This is an open access article distributed under the terms of the Creative Commons Attribution License (https://creativecommons.org/licenses/by/4.0/) which permits unrestricted use, distribution, and reproduction in any medium, provided the original author and source are credited.

\section{Introduction}

Acute kidney injury (AKI) and Chronic kidney disease (CKD) is a complex syndrome defined as a dysfunction of kidney caused by numerous etiologies. The prevalence of AKI and CKD raised upto $20 \%$ of hospitalized patients, AKI has a negative impact in patient outcomes in the short and long term, increasing the risk of in-hospital mortality, longer hospital stays, cardiovascular events, progression to chronic kidney disease, and long-term mortality. ${ }^{1-3}$ The AKI and CKD has significantly risk injury failure loss of kidney function -finally leads to end stage renal

\footnotetext{
* Corresponding author.

E-mail address: drumakoneti10@gmail.com (Uma K).
}

disease (ESRD). ${ }^{4}$ The pathophysiology of AKI and CKD multifactorial, hemodynamic instability, microcirculatory dysfunction, tubular cell injury, tubular obstruction, renal congestion. 5,6

The current definition of AKI was proposed by the Kidney Disease Improving Global Outcomes (KDIGO) work group in 2012. ${ }^{7}$ The KDIGO classification defines $\mathrm{AKI}$ as an increase in serum creatinine ( $\mathrm{SCr}$ ) of at least $0.3 \mathrm{mg} / \mathrm{dl}$ within $48 \mathrm{~h}$ and AKI in stages of severity which correlate with patient prognosis. The important biomarkers like Serum Creatinine and Urinary Albuminuria for detection of AKI and CKD and also these markers are altered by factors affectingits production (age, gender, diet, and muscle mass), elimination (previous renal dysfunction), 
and secretion (medications). ${ }^{8,9}$ Therefore, research has focused on the development of new biomarkers, including serum adiponectin, hemoglobin and RBCs. Adiponectin (ADPN) is an important protein that secrete by adipocytes. Plasma level of ADPN is suggested as a predictive marker of metabolic syndrome in patients with chronic kidney disease (CKD) who did not undergo dialysis. ${ }^{10}$ ADPN has both anti-inflammatory and anti-oxidative properties and deficiency causes foot processes effacement and albuminuria which are reverted after ADPN therapy. ${ }^{11}$ In some studies, plasma ADPN level is directly associated with cardiovascular diseases (CVD) and mortality in predialysis CKD patients and also other studies low plasma ADPN level predicts high cardiovascular events in HD patients. ${ }^{12}$ The present study was evaluated by a study serum adiponectin, hemoglobin and rbcs levels in patients with acute and chronic kidney diseases.

\section{Materials and Methods}

This is the case - control study was conducted in "Akash Institute of Medical Sciences and Research Centre", Karnataka from 2015-2020. A total 150 subjects included in the present study 100 Kidney diseases Patients (50AKI Patients and 50 CKD Patients) and 50 Healthy controls. All the subjects were recruited in the study after obtaining their informed consent after obtaining of ethical clearance from the institute (IEC No -164). Patients with age more than 30 years along with those patients diagnosed with AKI and CKD were included and Exclusions criteria were the presence of active infection, acute myocardial infarction in last 6 weeks, liver and pancreatic disorder, uncontrolled diabetes mellitus consumption of antioxidant drugs, and presence of active inflammatory diseases. From the all subjects, after overnight fasting (12hrs), $6 \mathrm{ml}$ of venous blood was collected and transferred, $3 \mathrm{~mL}$ into anticoagulant (EDTA) Tube and $3 \mathrm{~mL}$ into plain tube along with that urine samples also collected. The Total count and Urinary Albuminuria was processed immediately and plain serum were separated by centrifugation at $4000 \mathrm{rpm}$ for $3 \mathrm{~min}$ and stored until biochemical analysis was done.

Serum Urea, Creatinine and Uric Acid levels was analysed by using Commercially available laboratory standard methods. The total count like Hemoglobin, RBCs, was analysed by Sysmex KN-21N (manufactured by Sysmex Corporation Kobe, Japan) and the serum Adiponectin levels was analysed by using Enzyme Linked Immunoassay Method, Urinary Albuminuria was analysed by Albumin and Creatinine Ratio.

\subsection{Statistical analysis}

The normal distribution of data checked by using Kolmogorov Smirnov test. All the characters descriptively summarized. The mean and standard deviation about the arithmetic mean were used. The Variations in the serum Adiponectin and hemoglobin, RBCs was analysed by using Student's T-Tests (2 Tailed). The analysis of variance (ANOVO) followed by posthoc analysis done in between different groups. The correlation between the serum Adiponectin and Hemoglobin, RBCs, Urinary Albuminuria was done by using Pearson Correlation analysis. The Data was compiled in Microsoft excel spread sheets and analyzed using SPSS for windows version 16.0. A $p$ value $<0.05$ was considered statistically significant.

\section{Results}

Table 1 shows the data distribution of healthy controls and two groups of kidney diseases (AKI \& CKD) patients by using Kolmogorov Smirnov Test. All the parameters studiedhad at least one group with not normally distributed data. Hence, data was logarithmicallytransformed before applying parametric statistical tools.

Table 2 shows the demographic and clinical characteristics of the healthy controls and two groups of acute and chronic kidney disease subjects, The Serum urea, Creatinine, Uric Acid, Hemoglobin, RBCS and Serum Adiponectin, UrinaryAlbuminuria mean levels statistically significant difference between two groups of two groups of acute and chronic kidney disease patients when compared to healthy controls (P-0.0001**) by using independent sample ( 2 tailed) T- Test.

Table 3 shows the demographic and clinical characteristics of the different groups of study subjects, The hemoglobin and RBCs levels significantly reduced in two groups acute and chronic kidney disease patients when compared to healthy controls ( P- $0.0001 * \& 0.037 *$ ). The Serum urea, Creatinine, Uric Acid, Adiponectin and Urinary Albuminuria levels are elevated acute and chronic kidney disease patients when compared to healthy subjects by using analysis of variance (ANOVO) followed by post hoc analysis ( $\left.\mathrm{P}-0.0001^{*}\right)$.

Table 4 shows the positive correlation of Serum Adiponectin and Serum Urea, Creatinine, Uric Acid, Hemoglobin, RBCs and Urinary Albuminuria $(\mathrm{r}=0.834 * *$, $\left.0.866^{* *}, 0.754^{* *}, 0.715^{* *}, 0.732^{* *}, \mathrm{P}-0.0001\right)$ and negative correlation with the RBCs $(r=0.114, \mathrm{P}-0.129)$ in patients with groups acute and chronic kidney disease.

\section{Discussion}

Adipose tissue is a storage organ in the body and also act as a endocrine tissue that secrets several biological compounds like cytokines and peptide hormones which is known as a adipokines. ${ }^{13}$ This adipokines are involves in physiological functions like regulates metabolism and inflammation in some of the chronic diseases. ${ }^{14}$ The adiponectin is also one of the adipocytokine has anti- inflammatory, antiatherogenic and anti- diabetic properties, altered levels of 
Table 1: Assessment of distribution of data using Kolmogorov-Smirnov Test

\begin{tabular}{|c|c|c|c|}
\hline Parameter & Group $1(n=50)$ Controls & Group $2(n=50)$ & Group $3(n=50)$ \\
\hline Age & 0.076 & $0.200 *$ & 0.0001 \\
\hline Serum Urea & 0.021 & 0.0001 & $0.200 *$ \\
\hline Serum Creatinine & 0.001 & 0.068 & 0.022 \\
\hline Serum Uric Acid & 0.009 & 0.074 & 0.060 \\
\hline Urinary Albuminuria & 0.0001 & 0.0001 & 0.001 \\
\hline Hemoglobin & $0.200^{*}$ & 0.091 & 0.077 \\
\hline $\mathrm{RBCs}$ & $0.200^{*}$ & 0.004 & $0.200^{*}$ \\
\hline Serum Adiponectin & $0.200 *$ & $0.200^{*}$ & 0.028 \\
\hline
\end{tabular}

Table 2: Demographic characteristics and biochemical parameters studied in controls and two group's acute and chronic kidney disease patients by Independent Samples Test.

\begin{tabular}{|c|c|c|c|}
\hline Parameter & Controls $(n=50)$ & Cases $(n=100)$ & $P$ - Value $(n=50)$ \\
\hline Age & $47.93 \pm 13.68$ & $50.59 \pm 11.19$ & 0.173 \\
\hline Serum Urea & $20.07 \pm 5.71$ & \pm 18.79 & $0.0001 * *$ \\
\hline Serum Creatinine & $0.73 \pm 0.17$ & $9.03 \pm 5.29$ & $0.0001 * *$ \\
\hline Serum Uric Acid & $4.58 \pm 1.26$ & $7.78 \pm 1.25$ & $0.0001 * *$ \\
\hline Urinary Albuminuria & $9.85 \pm 0.95$ & $87.32 \pm 63.08$ & $0.0001 * *$ \\
\hline Hemoglobin & $13.65 \pm 2.00$ & $9.04 \pm 2.37$ & $0.0001 * *$ \\
\hline $\mathrm{RBCs}$ & $4.69 \pm 1.28$ & $4.29 \pm 0.54$ & $0.003^{*}$ \\
\hline Serum Adiponectin & $3.57 \pm 0.94$ & $11.89 \pm 4.95$ & $0.0001 * *$ \\
\hline
\end{tabular}

Table 3: Demographic characteristics and biochemical parameters studied in controls and three groups of acute and chronic kidney disease patients

\begin{tabular}{lcccc}
\hline Parameter & Group 1 $(\mathbf{n = 5 0})$ & Group 2 $(\mathbf{n = 5 0})$ & Group 3 $(\mathbf{n = 5 0})$ & p- Value \\
Age & $47.93 \pm 13.68$ & $51.38 \pm 11.49$ & $49.70 \pm 10.92$ & 0.297 \\
Serum Urea & $20.07 \pm 5.71$ & $59.23 \pm 10.55$ & $90.75 \pm 9.80$ & $0.0001^{* *}$ \\
Serum Creatinine & $0.73 \pm 0.17$ & $3.99 \pm 0.58$ & $14.11 \pm 1.98$ & $0.0001^{* *}$ \\
Serum Uric Acid & $4.58 \pm 1.26$ & $6.87 \pm 1.14$ & $8.70 \pm 0.41$ & $0.0001^{* *}$ \\
Urinary Albuminuria & $9.85 \pm 0.95$ & $48.87 \pm 46.60$ & $127.67 \pm 51.92$ & $0.0001^{* *}$ \\
Hemoglobin & $13.65 \pm 2.00$ & $10.64 \pm 2.37$ & $7.41 \pm 0.65$ & $0.0001^{* *}$ \\
RBCs & $4.69 \pm 1.28$ & $4.18 \pm 0.58$ & $4.39 \pm 0.48$ & $0.006^{*}$ \\
Serum Adiponectin & $3.57 \pm 0.94$ & $8.09 \pm 1.32$ & $15.81 \pm 4.16$ & $0.0001^{* *}$ \\
\hline
\end{tabular}

Table 4: Pearson Correlation in between serum Adiponectin with Serum Urea, Creatinine, Uric Acid, Hemoglobin, RBCs and Urinary Albuminuria

\begin{tabular}{|c|c|c|c|}
\hline \multirow{7}{*}{$\begin{array}{l}\text { Serum } \\
\text { Adiponectin }\end{array}$} & Parameter & r value & P value \\
\hline & Serum Urea & $0.834 * *$ & 0.0001 \\
\hline & Serum Creatinine & $0.866 * *$ & 0.0001 \\
\hline & Serum Uric Acid & $0.754 * *$ & 0.0001 \\
\hline & Urinary Albuminuria & $0.715 * *$ & 0.0001 \\
\hline & Hemoglobin & $0.732 * *$ & 0.0001 \\
\hline & $\mathrm{RBCs}$ & 0.114 & 0.129 \\
\hline
\end{tabular}

adiponectin strongly correlated with oxidative stress and urinary microalbuminuria, leads to Metabolic Syndrome and acute $\&$ chronic kidney diseases. ${ }^{15,16}$

Acute kidney injury (AKI) and Chronic kidney disease (CKD) is a complex syndrome defined as a dysfunction of kidney due to increased oxidative stress levels, urinary microalbuminuria gold standard method for early detection and progression of acute and chronic kidney diseases along with the glomerular filtration rate (GFR) and some of the recent studys found that urinary microalbuminuria is not a specific and sensitive method for detectionbecause it is elevated in other conditions like diabetic nephropathy, hypertension and other inflammatory conditions. ${ }^{17-19}$ Recent study found adiponectin is the specific and sensitive biomarker for early detection of AKI and CKD. The present study also significantly increased levels of serum adiponectin levels was observed in both the acute and chronic kidney diseases and compared with 
the healthy controls. Similar results previous studys also found that significantly elevated serum adiponectin levels were triggers anti-oxidative pathways through AMP kinase; NADPH enzymatic activity results reduce the infiltration of albuminuria from the kidney. ${ }^{20,21}$

The relationship between AKI, CKD and Anemia is conflict. This two disorders contribute to the development of anemia results increased risk of bleeding and decrease life span of red blood cells in the spleen. ${ }^{22}$ The cyclic pattern of hemoglobin levels stimulated by Both pharmacologic features and dosing of erythropoiesis-stimulatingagents and pathological variations of low and high hemoglobin levels areassociated with increased cardiovascular events and death in patients with AKI and CKD. ${ }^{23}$ The present study also observed that decreased levels of hemoglobin as well as Red Blood Cells in patients with both acute and chronic kidney diseases when compared to healthy controls. Hemoglobin and RBCs variability include acute or chronic comorbidities alters in iron stores, inflammation, blood loss ortransfusion, dialysis treatment featuressuch as dialysis adequacy, water quality, stage of CKD and residual renal function, level of parathyroid hormones and vitamin D, B12, or folate deficiencies. This all are leads to decreased hemoglobin and red blood cells levels in patients with acute and chronic kidney diseases leads to anemia. ${ }^{24}$

The present study also found the serum adiponectin levels are positively correlates with serum urea, creatinine, uric acid and urinary microalbuminuria levels and negatively correlates with hemoglobin and red blood cells in two groups acute and chronic kidney diseases when compared to healthy controls. Similarly previous studys also reported that the increased serum adiponectin levels are useful for early detection of acute and chronic kidney diseases than urinary microalbuminuria.

\section{Conclusion}

In conclusion, to estimate serum levels of adiponectin and hemoglobin, RBCs are useful for early detection and monitoring of disease progression in patients with acute and chronic kidney diseases.

\section{Source of Funding}

No financial support was received for the work within this manuscript.

\section{Conflict of Interest}

The authors declare they have no conflict of interest.

\section{References}

1. Stefan N. Plasma adiponectin concentrations in children: relationships with obesity and insulinemia. $J$ Clin Endocrinol Metab. 2002;87(10):4652-6.

2. Mojiminiyi OA, Abdella NA, Arouj MA, Nakhi AB. Adiponectin, insulin resistance and clinical expression of the metabolic syndrome in patients with Type 2 diabetes. Int J Obes. 2007:31(2):213-20. doi:10.1038/sj.ijo.0803355,

3. Monzillo LU, Hamdy O, Horton ES, Ledbury S, Mullooly C, Jarema $\mathrm{C}$, et al. Effect of Lifestyle Modification on Adipokine Levels in Obese Subjects with Insulin Resistance. Obes Res. 2003;11(9):104854. do1:10.1038/oby.2003.144

4. Onat A, Hergenc G, Dursunovglu D, uc ZK, Bulur S. Relatively high levels of serum adiponectin in obese women, a potential indicator of anti-inflammatory dysfunction: relation to sex hormone-binding globulin. Int J Biol Sci. 2008;4(4):208-14.

5. Axelsson J, urger OH, Stenvinkel P. Adipose tissue and inflammation in chronic kidney disease. Contributions Nephrol. 2006;151:165-74.

6. Hoste EAJ, Bagshaw SM, Bellomo R, Cely CM, Colman R, Cruz $\mathrm{DN}$, et al. Epidemiology of acute kidney injury in critically ill patients: the multinational AKI-EPI study. Intensive Care Medicine. 2015;41(8):1411-1423. Available from: https://dx.doi.org/10.1007/ s00134-015-3934-7. 100:10.1007/s00134-015-3934-7.

7. Mandelbaum T, Scott DJ, Lee J, Mark RG, Malhotra A, Waikar SS, et al. Outcome of critically ill patients with acute kidney injury using the Acute Kidney Injury Network criteria*. Critical Care Medicine. 2011;39(12):2659-2664. Available from: https://dx.doi.org/10.1097/

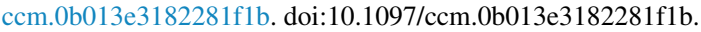

8. Kidney Disease: Improving Global Outcomes (KDIGO) Acute Kidney Injury Work Group. KDIGO clinical practice guideline for acute kidney injury. Kidney Int. 2012;2:1-138.

9. Shema-Didi L, Ore L, Geron R, Kristal B. Is Anemia at Hospital Admission Associated with In-Hospital Acute Kidney Injury Occurrence? Nephron Clin Pract. 2010;115(2):c168-76.

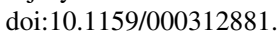

10. Halvorsen Y, Wilkison WO, Briggs MR. Human adipocyte proteomics - a complementary way of looking at fat. Pharmacogenomics.

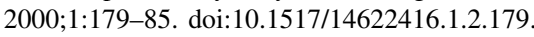

11. Huang JW, Yen CJ, Chiang HW. Adiponectin in peritoneal dialysis patients: a comparison with hemodialysis patients and subjects with normal renal function. Am J Kidney Dis. 2004;43:1047-55.

12. Staiger H, Tschritter O, Machann J, Thamer C, Fritsche A, Maerker E, et al. Relationship of Serum Adiponectin and Leptin Concentrations with Body Fat Distribution in Humans. Obes Res. 2003;11(3):368-72. doi:10.1038/oby.2003.48.

13. Sweiss N, Sharma K. Adiponectin effects on the kidney. Best Pract Res Clin Endocrinol Metab. 2014;28(1):71-9. 001:10.1016/..beem.2013.08.002

14. Trimarchi H, Muryan A, Dicugno M, Forrester M, Lombi F, Young $\mathrm{P}$, et al. In hemodialysis, adiponectin, and pro-brain natriuretic peptide levels may be subjected to variations in body mass index. Hemodialysis Int. 2011;15(4):477-84. doi:10.111/j.1542 14758200100562.

15. Ho KJ, Xue H, Mauro CR, Nguyen B, Yu P, Tao M, et al. Impact of uremia on human adipose tissue phenotype. J Surg Res. 2013;179(1):175-82. do1:10.1016/].]SS.2012.08.043.

16. Kamimura MA, Canziani MF, Sanches FR, Velludo CM, Carrero JJ, Bazanelli AP, et al. Variations in adiponectin levels in patients with chronic kidney disease: a prospective study of 12 months. J Bras Nefrol. 2012;34(3):259-65. 100:10.5935/0101-2800.20120007.

17. Okuno S, Ishimura E, Norimine K, Tsuboniwa N, Kagitani S, Yamakawa K, et al. Serum adiponectin and bone mineral density in male hemodialysis patients. Osteoporos Int. 2012;23(7):2027-35.

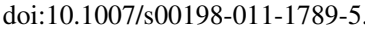

18. Hara K, Uchida T, Takebayashi K, Sakai Y, Inoue T, Inukai T, et al. Determinants of Serum High Molecular Weight (HMW) Adiponectin Levels in Patients with Coronary Artery Disease: Associations with Cardio-renal-anemia Syndrome. Int Med. 2011;50(24):2953-60. doi:102169/internalmedicine.50.5926

19. Kollerits B, Fliser D, Heid IM, Ritz E, Kronenberg F. Mmkd Study Group . Gender-specific association of adiponectin as a predictor of progression of chronic kidney disease: the Mild to ModerateKidney Disease Study. Kidney Int. 2007;71(12):1279-86.

20. Zhu W, Cheng KKY, Vanhoutte PM, Lam KSL, Xu A. Vascular effects of adiponectin: molecular mechanisms and potential therapeutic 
intervention. Clin Sci. 2008;114(5):361-74. 001:0.1042/cs20070347

21. Hughes JT. Associations of serum adiponectin with markers of cardio-metabolic disease risk in Indigenous Australian adultswith good health, diabetes and chronic kidney disease. Obes Res Clin Pract. 2016;10(6):659-72. doi:10.1016/1.orcp.2015.11.008.

22. Thongprayoon C, Cheungpasitporn W, Podboy AJ, Gillaspie EA, Greason KL, Kashani KB, et al. The effects of contrast media volume on acute kidney injury after transcatheter aortic valve replacement: a systematic review and meta-analysis. J Evid Based Med. 2016;9(4):188-93. doi:10.1111/jebm.12208

23. Karkouti K, Grocott HP, Hall R, Jessen ME, Kruger C, Lerner AB, et al. Interrelationship of preoperative anemia, intraoperative anemia, and red blood cell transfusion as potentially modifiable risk factors for acute kidney injury in cardiac surgery: a historical multicentre cohort

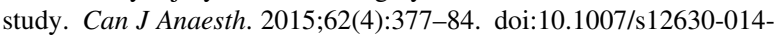
0302-y.
24. Loor G, Rajeswaran J, Li L, Sabik JF, Blackstone EH, McCrae KR, et al. The least of 3 evils: Exposure to red blood cell transfusion, anemia, or both? J Thorac Cardiovasc Surg. 2013;146(6):1480-7. doi:10.1016/1.jtcvs.2013.06.033

\section{Author biography}

Anil Malleshi Betigeri, Associate Professor

Uma K, Assistant Professor

Cite this article: Betigeri AM, Uma K. A Study of levels of serum Adiponectin, Hemoglobin and RBCs in patients with acute and chronic kidney diseases. IP J Diagn Pathol Oncol 2021;6(1):38-42. 